\title{
PERPENDICULAR AND PARALLEL TRANSPORT, AND INTERLAYER EXCHANGE COUPLING IN MAGNETIC TRILAYERS WITH EXTRA CAP-LAYERS
}

\author{
S. KROMPIEWSKI AND M. ZWIERZYCKI \\ Institute of Molecular Physics, Polish Academy of Sciences \\ Smoluchowskiego 17, 60-179 Poznań, Poland
}

\begin{abstract}
Oscillations of magnetoresistance and interlayer exchange coupling vs. both non-magnetic spacer and cap-layer thicknesses are studied within the framework of the $s-d$ model. The studies are carried out by means of the Kubo formula and a Green function recursion technique. Transport calculations concern multilayers (sandwiched between two semi-infinite ideal lead wires) oriented either parallel or perpendicular to the current direction. The considered structures for the above mentioned two basic geometries, are cubic grains identical in: (i) size, (ii) number of sublayers, and (iii) thicknesses of particular sublayers, which makes it possible to compare, in a direct and reliable way, the corresponding magnetoresistances. The current-in-plane magnetoresistance is found to be usually lower than the current-perpendicular-to-plane one, but both of them show oscillatory behaviours mutually correlated, and related to the Fermi surface callipers. Moreover, the current-perpendicular-to-plane giant magnetoresistance for structures in a form of infinite in layer-plane trilayers capped with an extra bilayer (ferromagnet/paramagnet) is also discussed.
\end{abstract}

PACS numbers: 75.70.-i, 75.70.Pa, 73.23.Ad

\section{Introduction}

Transport properties of magnetic multilayers have been intensively studied for a decade now. The interest is motivated by potential (and already partially realized) practical applications based first of all on the giant magnetoresistance (GMR) phenomenon [1-3]. The most common geometries in which the GMR is usually measured and calculated are CIP (current-in-plane) and CPP (current-perpendicular-to-plane), although the intermediate case CAP (current-at an angle-to-plane) is also tractable. In this paper we shall concentrate on CIPand CPP-GMR in order to elucidate the nature of these phenomena and their relation to interlayer exchange coupling (IEC). Unlike other authors, we construct 
our structures in a form of cubic grains and compare CIP and CPP results for samples with the same sizes and thicknesses of particular sublayers. Moreover, we carry out our considerations not only for varying spacer thicknesses but for varying cap-layer thicknesses, too.

The aim of the present paper is to compute the GMR, within the ballistic regime, for the two above-mentioned geometries, by means of an exactly solvable $s-d$ model with sublayer-, orbital- and spin-dependent on-site potentials. This approach, free from hardly controllable factors (like structural imperfections or thermal excitations) makes it possible to treat CIP and CPP on exactly equal footing.

\section{Model and formalism}

A "semi-realistic" $s-d$ model we adopt, has proved to work quite well in dealing with magnetoconductance problems in $\mathrm{Cu} / \mathrm{Co}$ multilayers (refer [4] for more details, including parameters: $t_{s}=-1, t_{d}=-0.2$ and $V_{s d}=1$, i.e. hopping integrals and hybridization). In the present study however a real-space representation is used and finite cubic magnetic layered structures are sandwiched between semi-infinite ideal lead wires (LW) made of the same metal as the paramagnetic spacer (i.e. with the same parameters, including $V_{s d}$ ).

In linear response theory, the conductance $\Gamma_{\sigma}$, i.e. the contribution of electrons with spin $\sigma$ to the dc-conductance reads

$$
\Gamma_{\sigma}=\frac{4 e^{2}}{h} \operatorname{Tr}\left[\tilde{G}_{\sigma}(0,0) \hat{T} \tilde{G}_{\sigma}(1,1) \hat{T}-\hat{T} \tilde{G}_{\sigma}(1,0) \hat{T} \tilde{G}_{\sigma}(1,0)\right],
$$

where 0 and 1 stand for indices of any neighbouring monolayers normal to the current direction, $\hat{T}$ is the diagonal hopping matrix, $\tilde{G}$ denotes the imaginary part of the Green function and the trace $\operatorname{Tr}[\ldots]$ is performed over both the orbitals $s, d$ as well as the lattice-sites within the given plane. Finally, the GMR is given by GMR $=\left(\Gamma_{\uparrow}^{\uparrow \uparrow}+\Gamma_{\downarrow}^{\uparrow \uparrow}\right) /\left(\Gamma_{\uparrow}^{\uparrow \downarrow}+\Gamma_{\downarrow}^{\uparrow \downarrow}\right)-1$, where the superscript $\uparrow \uparrow(\uparrow \downarrow)$ denotes the parallel (antiparallel) alignment of magnetic sublayers.

In addition to the transport phenomena we are interested in the mutual correlations between the GMR and the interlayer exchange coupling $(J)$. The $J$ has been determined from the configuration- and spin-dependent grand thermodynamic potentials (cf. [4]). The results we present below have been obtained for the following spacer $(S)$, ferromagnet $(F)$ and cap-layer $(C)$ on-site potentials: $\epsilon_{i \sigma}^{s}=0$ and $\epsilon_{i \uparrow}^{d}=-1$ for $i \in S$ and $F, \epsilon_{i \downarrow}^{d}=-1$ for $i \in S, \epsilon_{i \downarrow}^{d}=-0.2$ for $i \in F$, and $\epsilon_{i \sigma}^{d}=-0.2$ for $i \in C$ (all normalized to $\left|t_{s}\right|$ ), and Fermi energy $E_{\mathrm{F}}=0$.

\section{Discussion}

The main results of the present paper are presented in Figs. 1 and 2. All the depicted curves have oscillatory character with short and long periods. The periods of the IEC may be asymptotically estimated, from energy spectra, either for thick spacer limit or for thick "cap-layer limit. For the parameters under consideration, the so estimated periods are 2.8 and 8.2 in the former case (varying $n_{s}$ ) and 3.4 and 6.8 in the latter case (varying $n_{\text {cap }}$ ). As can be seen from the figures the GMR curves show also two types of periods and it depends on the geometry 
(CIP or CPP) and the varying layer $(S$ or $C$ ) whether the dominant period is long or short. Incidently the periods which appear in Figs. 1 and 2 are roughly consistent with the asymptotic ones mentioned above. Besides it is noteworthy that in the GMR vs. $n_{s}$ case the CPP curve lies always higher than the CIP curve in concordance with other papers (e.g. [5-7]). The GMR vs. $n_{\text {cap }}$ case has not been studied so far, to our knowledge, the interesting finding about it is that then the CIP and CPP curves are no longer very distant from each other and, first of all, that for specific $n_{\text {cap }}$-thicknesses (dependent also on $n_{s}$ ) the GMR can be brought to negative values corresponding to the so-called inverse GMR $[8,9]$.
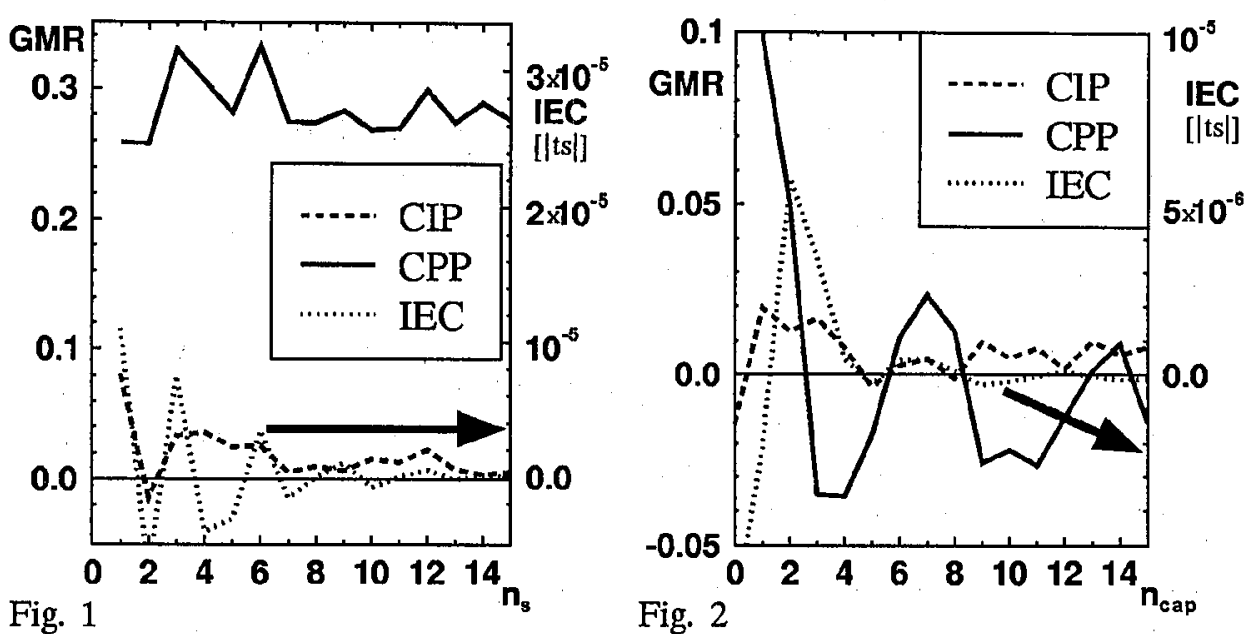

Fig. 1. GMR for the trilayer $n_{\text {cap }} / n_{f} / n_{s} / n_{f}$ with $n_{\text {cap }}=0, n_{f}=3$, vs. spacer thickness $\left(n_{s}\right)$. The CPP-GMR is much larger than the CIP-GMR, the former reveals predominantly short-period oscillations whereas the latter has got a quite pronounced long period oscillation component. The dotted curve represents interlayer exchange coupling (right hand side scale). All the thicknesses are expressed in the number of monolayers. Fig. 2. As in Fig. 1 but vs. cap-layer thickness and $n_{s}=2$. This time the long-range oscillations dominate for the CPP case, whereas the short-range ones - for the CIP case.

We present also results for the CPP geometry with a bit more complex structure of the form $F_{1} / S_{1} / F_{2} / S_{2} / F_{3}$ (infinite in layer-plane dimensions), i.e. a spin-valve with the $F_{1} / F_{2}$ antiferromagnetic exchange coupling stronger than that of $F_{2} / F_{3}$. Formally this structure may be regarded as the trilayer capped with $C=F_{1} / S_{1}$ bilayer [10]. It is easily seen from Fig. 3 that IEC (between $F_{2}$ and $F_{3}$ ) and GMR remain correlated again.

In conclusion, it has been shown that the GMR exhibits oscillatory behaviour with quasiperiods determined by the Fermi energy callipers. Within the ballistic regime, the GMR reveals clearly multiperiodic behaviour but weights of particular periods are different, so some periods may be quite pronounced whereas others - substantially suppressed. For instance, according to the present model, the 


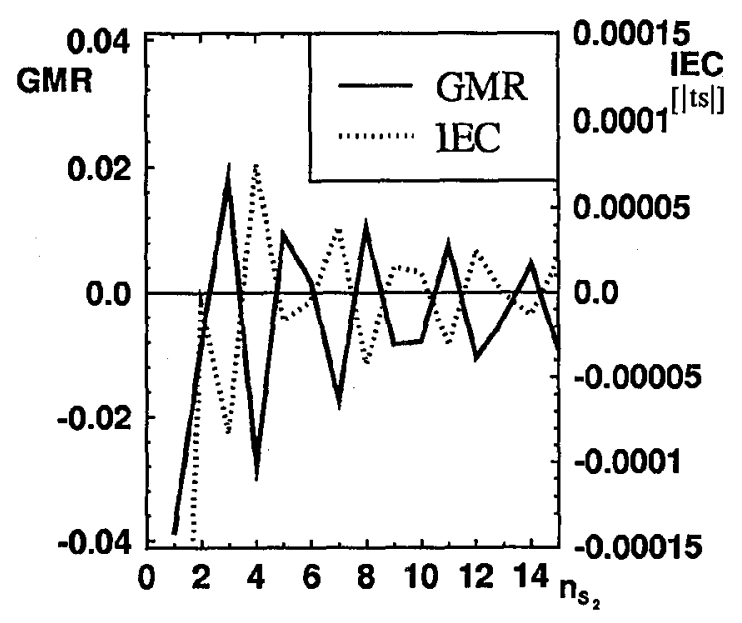

Fig. 3. GMR and IEC for the $F_{1} / S_{1} / F_{2} / S_{2} / F_{3}$ spin-valve with the thicknesses equal to 8,5 , and 3 for magnetic sublayers and 3 for the first spacer $\left(S_{1}\right)$.

long period of the GMR is clearly visible both for the CIP geometry with varying spacer-thickness as well as for the CPP geometry with varying cap-layer-thickness, whereas the short period dominates in the alternative cases (CIP-GMR vs. $n_{\text {cap }}$ and CPP-GMR vs. $n_{s}$ ). The correlation between CPP-GMR and interlayer exchange coupling is still strong in case of the spin-valve structure.

\section{Acknowledgments}

We gratefully acknowledge the grants No. 2P03B-118-14 and 2P03B-099-11 of the Committee for Scientific Research, and thank the Poznan Supercomputing and Networking Center for the computing time.

\section{References}

[1] G.A. Prinz, Physics Today 48, 58 (1995).

[2] Z. Wang, Y. Nakamura, J. Magn. Magn. Mater. 155, 161 (1996).

[3] J.M. Daughton, J. Magn. Magn. Mater. 192, 334 (1999).

[4] S. Krompiewski, U. Krey, Europhys. Lett. 44, 661 (1998).

[5] S. Zhang, P.M. Levy, J. Appl. Phys. 69, 4786 (1991).

[6] Y. Asano, A. Oguri, S. Maekawa, Phys. Rev. B 48, 6192 (1993).

[7] K.M. Schep, P.J. Kelly, G.E.W. Bauer, Phys. Rev. Lett. 74, 586 (1995).

[8] J,-P. Renard, P. Bruno, R. Megy, B. Bartenlian, P. Beauvillain, C. Chappert, C. Dupas, E. Kolb, M. Mulloy, J. Prieur, P. Veillet, E. Velu, J. Appl. Phys. 79, $5270(1996)$.

[9] J. Binder, P. Zahn, I. Mertig, J. Magn. Magn. Mater, 165, 100 (1997).

[10] M. Zwierzycki, S. Krompiewski, J. Magn. Magn. Mater. 202, 150 (1999). 